\title{
Poderes Ejecutivos y derecho al aborto bajo gobiernos progresistas en América Latina: Ciudad de México y Brasil*
}

Executive Powers and the Right to Abortion under Progressive Governments in Latin America: Mexico City and Brazil

\author{
ALBA RUIBAL** \\ Universidad Nacional de Córdoba, Argentina \\ Consejo Nacional de Investigaciones Científicas y Técnicas, Argentina. \\ albaruibal@gmail.com
}

https://doi.org/10.46468/rsaap.15.2.A3

\begin{abstract}
Resumen: El llamado giro a la izquierda en América Latina coincidió con el inicio de una serie de reformas que liberalizaron las leyes de aborto en distintos países de la región. Al mismo tiempo, en diversos países con gobiernos progresistas no se produjeron cambios en este campo. Este artículo argumenta que, además de movimientos fuertes y partidos favorables en el gobierno, en los regímenes políticos latinoamericanos, marcados por un fuerte presidencialismo, así como por un extenso poder de los gobernadores en países federales, es necesario analizar el rol de poderes ejecutivos y, en particular, los contextos pre y post electorales como coyunturas críticas que pueden generar incentivos para el avance o bloqueo de cambios legislativos en materia de aborto. Asimismo, se analiza la relación Estado-Iglesia como factor contextual significativo para reformas en esta área de derechos. Con base en investigación empírica, se estudian en particular los casos de la Ciudad de México y Brasil, que son contrastantes en términos del proceso de secularización, así como de resultados legislativos, y permiten señalar el papel de los incentivos electorales en el comportamiento de los Ejecutivos con relación al derecho al aborto.
\end{abstract}

Palabras clave: Aborto - Poder Ejecutivo - Izquierda - México - Brasil

\begin{abstract}
The so-called Left turn in Latin America coincided with the beginning of a series of reforms that liberalized abortion laws in different countries of the region. At the same time, in several countries under progressive governments in that period, there was no change in this field. This article argues that, in addition to strong movements and favorable parties in power, in Latin American political regimes, marked by strong presidentialism, as well as by an extensive power of governors in federal countries, it is necessary to analyze the role of executive powers, and in particular pre and post electoral contexts, as critical junctures that can generate incentives for them to advance or block reforms in this field. Likewise, State-Church relationships are also analyzed as a significant contextual factor for reforms in this area of rights. Based on empirical research, the study analyzes in particular the cases of Mexico City and Brazil. These cases are contrasting in terms of the secularization process, as well as of legislative results, and allow to point out the role of electoral incentives in the behavior of the Executives regarding abortion rights.
\end{abstract}

Key Words: Abortion - Executive Power - Leftist governments - Mexico - Brazil

* Artículo recibido el 22 de diciembre de 2020 y aceptado para su publicación el 20 de julio de 2021.

** La autora agradece el aporte de dos evaluaciones anónimas a una versión previa de este artículo. 


\section{Introducción}

El periodo caracterizado como giro a la izquierda, por el cual hacia 2005 gobiernos progresistas, de izquierda o centroizquierda, gobernaban a la mayoría de la población latinoamericana (Friedman y Tabbush, 2020: 19), coincidió con el inicio de una serie de reformas que liberalizaron las leyes de aborto en distintos países de la región. Desde el año 2000, en la Ciudad de México se produjo un proceso de cambio gradual que culminó en la legalización de la interrupción voluntaria del embarazo durante el primer trimestre en 2007; en 2012, el Congreso uruguayo legalizó el aborto durante el primer trimestre; en 2017, el Congreso en Chile aprobó tres excepciones a la criminalización total del aborto que existía en el país, basadas en riesgo para la vida de la mujer, casos de violación e inviabilidad fetal; en 2019, el estado mexicano de Oaxaca legalizó el aborto durante el primer trimestre, y la Argentina lo hizo en $2020 .^{1}$

Estos cambios legales son aún insuficientes para una región que presenta algunas de las leyes sobre aborto más limitantes en el mundo, con una prohibición total de esta práctica en cuatro países (El Salvador, Honduras, Nicaragua y República Dominicana) y una mayoría de países en los que el aborto en todas las etapas del embarazo es considerado un crimen, con ciertas excepciones o indicaciones (generalmente en casos de violación o riesgo para la vida o salud de la mujer). Sin embargo, las reformas recientes marcan un giro significativo con respecto a décadas de inacción en este campo en América Latina. A principios del siglo XX, diversos países de la región incluyeron excepciones a la criminalización, lo cual los situó como "vanguardias en el campo de la ley del aborto" (Htun, 2003: 143). Pero desde entonces, y hasta inicios del siglo XXI, en general no se ampliaron esos marcos legales. Menos aún se avanzó en permitir el aborto a demanda de la mujer durante el primer trimestre -como ocurrió en países europeos y norteamericanos durante las décadas de 1960 y 1970 (Rubio Marin, 2014)-, que hasta 2007 fue legal sólo en los casos especiales de Cuba (1965), Puerto Rico (1973) y Guyana (1995). Esto fue así a pesar de la presencia, desde los procesos de democratización, de fuertes movimientos feministas que tienen en su centro la demanda por el

Además de estos cambios legislativos, las cortes constitucionales latinoamericanas también tuvieron un papel relevante en el proceso de reformas ocurrido durante las dos últimas décadas en el campo del derecho al aborto en la región. En 2006, la Corte Constitucional colombiana estableció un modelo de indicaciones por razón de riesgo a la salud de la mujer, violación y malformación fetal incompatible con la vida; en 2012, el Supremo Tribunal Federal de Brasil incluyó una nueva causal para casos de anencefalia. En 2012 la Corte Suprema argentina, y en 2021 la Corte Constitucional de Ecuador, establecieron que el aborto es legal en todos los casos de violación. 
derecho al aborto -los cuales han sido el principal motor del avance en materia de derechos reproductivos en América Latina (Lamas, 2009; Reuterswärd et al., 2011; Lopreite, 2014; Tabbush et al., 2020)- y de que a partir de las transiciones a la democracia se realizaron cambios en prácticamente todas las áreas de los derechos de las mujeres en la región.

Frente a la parálisis que tuvo lugar durante décadas en América Latina en materia de derecho al aborto, la política legislativa en este campo durante las dos últimas décadas sugiere que la presencia de gobiernos progresistas ha sido un factor relevante para la realización de reformas. Con excepción de la despenalización del aborto en Oaxaca en 2019, que se produjo bajo el gobierno del Partido Revolucionario Institucional (PRI), los otros cuatro procesos de cambios legislativos en este campo -en Ciudad de México, Uruguay, Chile y Argentina- tuvieron lugar bajo gobiernos de izquierda o centroizquierda. Asimismo, más allá de la relevancia de estas reformas y del cambio que implican en la política del aborto en la región, se debe señalar también la falta de avances legislativos en este campo bajo una gran parte de gobiernos de izquierda -por ejemplo, Ecuador (2007-2017), Bolivia (20062019), Brasil (2003-2016), Argentina (2003-2015). Esta resistencia al cambio legal aún bajo un signo político más favorable es llamativa, dado que la socialdemocracia en el poder fue clave para la legalización del aborto a lo largo del continente europeo desde los años 1960s (Outshoorn, 1996), y que diversos partidos de esta orientación en América Latina han incorporado en sus plataformas los derechos reproductivos (por ejemplo, en los casos del Partido de la Revolución Democrática en México, el Partido de los Trabajadores en Brasil, y el Frente Amplio en Uruguay), o han tenido sectores importantes que han colocado el derecho al aborto en la agenda legislativa (por ejemplo, dentro del Movimiento Alianza PAIS en Ecuador).

Los estudios sobre política y género durante el llamado giro a la izquierda señalan que en ese periodo se produjeron avances significativos en las condiciones materiales de vida de las mujeres y hubo una amplia incorporación de mujeres en los cargos políticos, entre otras conquistas, pero al mismo tiempo en muchos casos existieron dificultades para avanzar en los temas más controvertidos de la agenda social, como el derecho al aborto (Zaremberg, 2016; Blofield, Ewig y Piscopo, 2017; Friedman y Tabbush, 2020). En este sentido, se ha señalado que, si bien la presencia de la izquierda en el poder ha sido un factor relevante para cambios significativos en materia de género, para dar cuenta de su rol en casos específicos y en determinadas áreas de derechos de las mujeres y de la diversidad sexual, es necesario analizar su interacción con otros factores políticos y sociales (Blofield, Ewig y Piscopo, 2017; Friedman y Tabbush, 2020). 
A través del estudio de dos procesos contrastantes en términos de resultados legislativos, en Brasil y la Ciudad de México, bajo gobiernos progresistas, este artículo se propone analizar el rol que han tenido los poderes ejecutivos en ambos casos y bajo qué condiciones han apoyado proyectos de reforma legislativa en materia del derecho al aborto, o se han negado a impulsarlos. Especialmente, se examina cómo ha incidido la política electoral, en tanto ventana de oportunidad o coyuntura crítica, en la generación de incentivos para que los Ejecutivos promovieran o no una reforma en este campo. Se señala en particular el modo en que un contexto pre-electoral en Brasil y un contexto post-electoral en México, incidieron en la política sobre el aborto. Asimismo, se considera en cada caso, como variable contextual, la relación entre el Estado y la Iglesia, que ha sido señalada como un factor central por la literatura sobre reformas en materia de derecho al aborto en América Latina (Htun, 2003; Lamas, 2009; Corrêa, 2010) y en otros contextos (Grzymała-Busse, 2015; Minkenberg, 2002). Brasil y en segundo lugar México son los dos países con mayor población católica en el mundo. Esto sugiere que no es la devoción religiosa de la población el factor que permite explicar el impacto de la interacción entre ambas esferas en el área del aborto, sino la tradición de separación entre la Iglesia y el Estado y los arreglos institucionales que la sostienen.

El trabajo no intenta ofrecer una explicación teórica general sobre el rol de estas variables, sino presentar un análisis contextualizado de las relaciones entre instituciones y resultados de igualdad de género, subrayando en particular el papel de los poderes ejecutivos, e incorporando los casos de Brasil y México, que generalmente no han sido analizados hasta ahora por la literatura sobre sobre política legislativa del aborto bajo gobiernos de izquierda en América Latina (Kampwirth, 2008; Reuterswärd et al., 2011; Blofield y Ewig, 2017; Viterna, 2012). Asimismo, si bien se considera especialmente el rol de los poderes ejecutivos en la posibilidad de reformas legislativas, se incluye también un análisis de su papel en la implementación del aborto legal, generalmente a través de órdenes ejecutivas y de los ministerios de salud.

Los casos de México y Brasil se analizan a partir de la literatura existente sobre el tema, así como de fuentes documentales, notas periodísticas y entrevistas llevadas a cabo por la autora en la Ciudad de México en 2011 y 2019, y en Brasil en 2012 y 2019. En la primera sección se presenta un panorama general sobre el rol de los poderes ejecutivos en procesos de reforma de las leyes de aborto en América Latina. En la segunda sección se analiza el proceso de legalización del aborto en la Ciudad de México, y en la tercera se analizan las dificultades para cambiar el marco legal del aborto bajo gobiernos progresistas en Brasil. 


\section{Ejecutivos y derecho al aborto en América Latina}

En los regímenes fuertemente presidencialistas latinoamericanos, caracterizados en algunos casos como hiperpresidencialismo (O’Donnell, 2011), los poderes ejecutivos han tenido históricamente amplias atribuciones constitucionales y una gran influencia en la formulación de políticas a nivel nacional. Como señala Lopreite, en países con un presidencialismo fuerte, jefes y jefas políticas poderosas tienen capacidad para disciplinar a sus bloques en el Congreso (2020: 221). De este modo, en estos sistemas de la región, los líderes políticos que ocupan la presidencia tienen una amplia capacidad de influir sobre las políticas públicas (Friedman y Tabbush, 2020: 56). De manera similar, entre los países federales de la región que se consideran casos de federalismo robusto -México, Brasil y Argentina-, los gobernadores disponen de importantes niveles de autonomía y recursos (Mainwaring, 1997: 27-28) y también tienen una amplia influencia sobre las políticas públicas locales. En estos regímenes, los gobernadores y jefes de gobierno de entidades federativas comprometidos con determinadas reformas pueden ser actores decisivos para efectuar cambios en el nivel subnacional, a pesar de las dificultades que hubiere para cambios similares en el nivel nacional (ver Mainwaring, 1997: 29). En el campo del derecho al aborto, México es el único país en la región, y uno de los pocos países del mundo, junto a Estados Unidos y Australia (Center for Reproductive Rights, 2014: 32), donde las leyes de aborto pueden determinarse en el nivel local, ya que los códigos penales y las leyes de salud son promulgadas por las legislaturas estatales.

La política reciente del aborto en la región muestra los diferentes roles que pueden desempeñar los poderes ejecutivos en procesos de reforma en este campo. En primer lugar, los Ejecutivos han promovido y apoyado directamente la legalización del aborto, o la liberalización de su marco legal, siendo actores centrales en el proceso de legalización del aborto en los casos de la Ciudad de México en 2007, bajo el gobierno Marcelo Ebrad, y en la Argentina en 2020, bajo el gobierno del Presidente Alberto Fernández. Asimismo, la Presidenta de Chile Michele Bachelet impulsó la ley que estableció la legalidad del aborto en tres causales en 2017, que fue redactada por el Poder Ejecutivo y negociada directamente en el Congreso por las ministras y asesoras de la presidencia. En segundo lugar, y en un extremo opuesto, los casos de Uruguay y Ecuador muestran cómo los Ejecutivos pueden convertirse en el obstáculo principal para la ampliación del derecho al aborto: el presidente Tabaré Vázquez usó sus poderes de veto contra el capítulo II de la Ley de Defensa a la Salud Sexual y Reproductiva que despenalizaba el aborto, impulsada por representantes de su propio partido y aprobada por 
el Congreso uruguayo en 2008. Un rol similar jugó el Presidente ecuatoriano Rafael Correa, quien frenó el proyecto de ley presentado por una mayoría de legisladores de su partido para legalizar el aborto en todos los casos de violación, durante el proceso de reforma del Código Penal concluido en 2014. En tercer lugar, Brasil durante el gobierno de Dilma Rousseff y Argentina durante los dos mandatos de Cristina Fernández son casos en los que no hubo un intento directo por parte de ambas Presidentas de obstruir el tratamiento legislativo del aborto, pero bajo ambas presidencias existió una voluntad manifiesta de no promover reformas en este sentido. En cuarto lugar, el caso argentino bajo el gobierno de Mauricio Macri, en 2018, muestra un Ejecutivo que inicialmente habilitó el debate legislativo sobre la legalización del aborto, pero posteriormente no apoyó la efectiva aprobación del proyecto de ley, que fue derrotado por un escaso margen en el Senado.

Las convicciones personales de quienes encabezan los poderes ejecutivos pueden ser altamente determinantes de su voluntad política con respecto especialmente a un área como el derecho al aborto, que es identificado por Htun y Weldon como un tema "doctrinario", en tanto interpela directamente creencias religiosas y tradiciones culturales (2010: 209). Ejemplos destacados en cuanto al rol de la posición ideológica personal de los Ejecutivos son el veto del Presidente Tabaré Vázquez en 2008 (ver Johnson, Rodríguez Gustá y Sempol, 2020), y la oposición sistemática del Presidente Rafael Correa a la liberalización de las leyes de aborto aduciendo motivos religiosos (Narváez, 2017). De un modo menos frontal y terminante, cuando era Presidenta Cristina Fernández expresó en distintas oportunidades que sus creencias religiosas estaban en la base de su oposición a la legalización del aborto $^{2}$-posteriormente, ella declararía haber cambiado su posición en este sentido, y como Senadora Nacional votó a favor de la legalización en 2018. Las convicciones personales del Ejecutivo también han sido importantes en sentido contrario, especialmente en el caso del Presidente argentino Alberto Fernández, quien antes de asumir su mandato se describió como un "activista de ponerle fin a la penalización del aborto". ${ }^{3}$

Sin embargo, más allá de las convicciones personales expresadas por los Ejecutivos y de su posición ideológica con relación al tema del aborto, en su decisión de apoyar o no una reforma pueden influir de manera prominente consideraciones estratégicas. En este sentido, las elecciones pueden confi-

2 "Di Tullio: 'El Congreso no está maduro para debatir la interrupción del embarazo", Infonews, 12 de mayo de 2014.

3 “Alberto Fernández enviará un proyecto de legalización del aborto al Congreso", Página 12, 17 de noviembre de 2019. 
guran coyunturas críticas, y en los contextos pre y post electorales los políticos pueden encontrar incentivos para promover $u$ obstaculizar el avance del derecho al aborto. Con base en el análisis de Tarrow (1996) sobre oportunidades políticas de los movimientos sociales, se puede plantear que la política electoral puede influir en los procesos de reforma de las leyes de aborto bajo gobiernos de izquierda de dos maneras principales. Por un lado, un contexto político post-electoral conflictivo, marcado por una alta polarización partidaria entre fuerzas de derecha e izquierda, puede crear incentivos para que los gobiernos de izquierda atiendan las demandas de los movimientos sociales progresistas a fin de diferenciarse de sus oponentes y fortalecer sus bases de apoyo y su poder político. En este sentido, como argumenta Tarrow, las divisiones entre las élites pueden crear ventanas de oportunidad para las demandas de los movimientos sociales, ya que pueden llevar a sectores de la élite a asumir el papel de "tribuno del pueblo" para obtener el apoyo de grupos sociales relevantes (1996: 56). El caso de la Ciudad de México ilustra cómo un contexto polarización post-electoral puede crear incentivos para que un Ejecutivo apoye la legalización del aborto.

Por otro lado, en contextos pre-electorales marcados por la incertidumbre electoral y la posibilidad de cambio en los alineamientos partidarios, los actores políticos pueden buscar el apoyo de fuentes externas al sistema político (Tarrow, 1996: 55). En América Latina, los actores e instituciones religiosas han sido históricamente uno de los poderes de facto cuyo apoyo ha buscado el poder político como fuente de legitimación externa. En estos casos, para obtener el apoyo de las fuerzas religiosas, incluso autoridades de izquierda pueden obstaculizar el avance del derecho al aborto, como ha ocurrido en países como Nicaragua y El Salvador (Kampwirth, 2008; Viterna, 2012), y como se muestra en este trabajo, también en el caso de Brasil.

El rol de los incentivos electorales en Nicaragua y el Salvador ha sido particularmente significativo, ya que partidos de izquierda, con una tradición anterior de apoyo a los derechos reproductivos, votaron o se pronunciaron a favor de leyes de aborto que están entre las más restrictivas del mundo. En Nicaragua, nueve días antes de la competida elección presidencial del 5 de noviembre de 2006, que ganaría Daniel Ortega, del Frente Sandinista de Liberación Nacional (FSLN), el Parlamento aprobó una reforma del código penal que eliminó la única excepción existente a la criminalización del aborto, por causa de riesgo a la vida de la mujer. El proyecto de ley fue impulsado por una alianza de iglesias católica y evangélicas, apoyada por los principales partidos, y fue aprobado por unanimidad por legisladores del FSLN, además de por representantes del partido conservador en el gobierno, a excepción del candidato de Renovación Sandinista. 
A pesar de no existir pruebas de un acuerdo del FSLN con los sectores conservadores, las estudiosas de este proceso señalan que, con esta reforma, el candidato presidencial del Frente Sandinista buscaba asegurar el apoyo de sectores religiosos que era decisivo para ganar la elección (Kampwirth, 2008; Reuterswärd et al., 2011). En El Salvador, ante la posibilidad de ganar las elecciones presidenciales de 2008, y durante una campaña en la cual el partido conservador ARENA utilizó el tema del aborto para caracterizar al Frente Farabundo Martí para la Liberación Nacional (FMLN) como un partido socialista radical, el ex líder guerrillero y entonces candidato presidencial por este último partido, Mauricio Funes, adoptó un fuerte discurso anti-aborto, que mantuvo durante su presidencia (2009-2014), permitiendo la aplicación de la draconiana ley del aborto que existe en el país desde 1997 (Viterna, 2012: 253). En ambos casos, un imperativo pragmático, la maximización de votos, desplazó a la dimensión ideológica o programática, poniendo de relieve el carácter instrumental que pueden tener para la dirigencia política las demandas feministas.

\section{Ciudad de México: polarización post-electoral y legalización del aborto}

México es el único país latinoamericano donde cada una de las entidades federativas (31 estados y la Ciudad de México) determinan sus códigos penales y civiles y, por lo tanto, es el único país en la región donde las leyes de aborto pueden ser promulgadas a nivel local. La oportunidad política para el avance del reclamo por el derecho al aborto en la Ciudad de México cambió en 1997, cuando sus residentes eligieron al Jefe de Gobierno de la Ciudad por votación popular por primera vez desde 1928, así como a los miembros de la Asamblea Legislativa unicameral reformada. Desde entonces, la Ciudad ha sido gobernada por la izquierda (el Partido de la Revolución Democrática, PRD, entre 1997 y 2018, y el Movimiento Regeneración Nacional, Morena, desde 2018), que también ha contado con mayoría en la Asamblea Legislativa. Asimismo, desde 1997, la Ciudad tiene su propio código penal y ley de salud, como las otras entidades federativas del país.

El proceso de reforma comenzó en el año 2000, cuando la Ciudad de México era una de las pocas entidades federativas cuya legislación sobre aborto no se había liberalizado desde la década de 1930, y tenía la ley más restrictiva del país (se incluían sólo excepciones por casos de violación, riesgo para la vida de la mujer y aborto accidental) (Lamas, 2009: 165). Desde ese año, se dio un proceso gradual de reformas legislativas que expandieron 
las condiciones bajo las cuales se permite la interrupción legal del embarazo en la Ciudad.

En primer lugar, el 18 de agosto de 2000, la Asamblea Legislativa local aprobó una reforma que las feministas habían exigido durante mucho tiempo (Lamas y Bissell, 2000), que incluyó tres excepciones adicionales a la criminalización del aborto, basadas en grave riesgo para la salud de la mujer, malformación fetal congénita, e inseminación artificial no consentida. Sin embargo, la nueva ley no estableció la obligación del estado de proporcionar servicios de aborto legal. En segundo lugar, el 26 de diciembre de 2003, debido a la demanda feminista en este sentido, la Asamblea Legislativa de la Ciudad aprobó una segunda reforma, que no cambió las indicaciones existentes, pero estableció, fundamentalmente que el aborto en los casos permitidos por la ley no debía considerarse un delito y que el sistema de salud pública de la Ciudad debía proporcionar servicios de aborto legal gratuitos. Como parte de la negociación política de la reforma, la nueva ley incorporó una cláusula de objeción de conciencia por primera vez en la ley de aborto en México (Cortés, 2006: 33), que había sido demandada por actores conservadores. De esta manera, en 2004 la Ciudad de México se convirtió en la jurisdicción con las normas y procedimientos de aborto más avanzados del país (Cortés, 2006: 33).

Pero fue en 2007 cuando la Ciudad dio el paso más importante y se convirtió en vanguardia en el campo del derecho al aborto en la región, al descriminalizar la interrupción del embarazo durante el primer trimestre y garantizar ampliamente el acceso a servicios de aborto legal (ver GIRE, 2008; Lamas, 2017; Ruibal, 2012). El 26 de abril de 2007, la Asamblea Legislativa del entonces Distrito Federal reformó el Código Penal y la Ley de Salud locales, y estableció tanto la interrupción legal del embarazo a demanda de la mujer durante las primeras doce semanas, como su provisión gratuita para las residentes de la Ciudad, y por un pequeño monto para residentes de otros estados, así como para mujeres extranjeras, en hospitales públicos bajo jurisdicción de la Ciudad. La ley de salud también declaró a la salud sexual y reproductiva como prioridad, y estableció que el gobierno debía implementar políticas públicas integrales para proporcionar información y orientación en esta materia, así como acceso a métodos de anticoncepción gratuitos. La reforma implicó un reconocimiento amplio de la autonomía de las mujeres, ya que no estipula que tengan que justificar su decisión ante ninguna autoridad.

Entre los tres principales partidos de México al momento de la reforma en 2007 (PRI, PRD y Partido Acción Nacional, PAN), el PRD es el que había mantenido la posición más sólida en términos de igualdad de género, y 
desde 1997 sus manifiestos electorales habían incluido la prestación de servicios de aborto legal entre sus propuestas en el campo de la salud de la mujer (Informante 8, entrevista de la autora 2011). Las organizaciones feministas apoyaron al PRD desde su creación en 1989, y la alianza del feminismo con este partido fue crucial en las reformas legales favorables a los derechos de las mujeres, incluido el aborto (Informante 1, entrevista de la autora 2011). Desde la primera reforma en el año 2000, las organizaciones feministas colaboraron con las autoridades de la Ciudad de México, proporcionando argumentos y apoyo técnico para las tres principales reformas a la ley de aborto de la Ciudad (Informante 10, entrevista de la autora 2011; Sánchez Fuentes et al., 2008: 350), así como para su implementación (Informante 13, entrevista de la autora 2011; Informante 12, entrevista de la autora 2019).

Aunque algunos sectores del PRD fueron más reacios a apoyar la legalización del aborto, el Jefe de Gobierno de la Ciudad en 2007, Marcelo Ebrard (2006-2012) adoptó el discurso de la ciudadanía como un principio clave de su gobierno y se presentaba como un político moderno (Informante 1, entrevista de la autora 2011). Durante su gobierno, Ebrard promovió un conjunto de reformas vanguardistas en materia de derechos individuales, que fueron aprobadas por la Asamblea Legislativa de la Ciudad, entre ellas, además de la despenalización del aborto, la primera ley del país sobre eutanasia pasiva (que permite la suspensión del tratamiento médico para los pacientes con enfermedad terminal) aprobada en 2008, y la ley sobre el matrimonio entre personas del mismo sexo, aprobada en 2009, que convirtió a la Ciudad de México en la primera jurisdicción en América Latina en legalizarlo.

Además de la presencia de un partido y un Jefe de Gobierno favorables al derecho al aborto en el gobierno local, que contaba con una mayoría en la Asamblea Legislativa de la Ciudad, el contexto de polarización política postelectoral contribuye a explicar la legalización del aborto en la Ciudad. Durante la campaña presidencial de 2006, la presencia de una candidata a Presidenta que provenía del feminismo -Patricia Mercado, del Partido Alternativa Socialdemócrata- colocó la cuestión del aborto en un lugar prominente de la discusión política, y los candidatos de los distintos partidos se vieron obligados a expresar su posición sobre el tema. Luego de que en las elecciones presidenciales el PAN, de centro derecha, con fuertes vínculos con la Iglesia Católica, ganara por una diferencia de 0,65 sobre el PRD, después de una campaña extremadamente polarizadora, con denuncias generalizadas de fraude electoral y una consecuente disputa sobre la legitimidad del nuevo Presidente de la República, la reforma del aborto ha sido 
considerada como una oportunidad para el PRD de señalar que su proyecto para el país difería del proyecto del PAN (Informantes 7 y 8, entrevistas de la autora 2011; Sánchez Fuentes et al., 2008: 353; Informante 12, entrevista de la autora 2019).

Como señalan Trejo y Ley (2016) -en su estudio sobre la variación geográfica en los niveles de violencia en las distintas entidades federativas del país-, la campaña presidencial de 2006 se convirtió en un momento clave de la confrontación ideológica sostenida entre derecha e izquierda en el México post-autoritario. Los autores observan que en esa confrontación el eje sociocultural era un tema fundamental del conflicto entre las dos fuerzas políticas antitéticas en esa confrontación: PAN y PRD, y que la Ciudad de México fue un enclave crucial en esa disputa (Trejo y Ley, 2016: 24), al llevar adelante proyectos que se oponían directamente a la visión del partido que gobernaba el país desde el año 2000 y que se consideró ganador de las elecciones en 2006.

De esta manera, el contexto de polarización post-electoral generado por las elecciones de 2006 favoreció la reforma, al crear incentivos para que un sector de la élite política, encabezado por el Jefe de Gobierno del entonces Distrito Federal, impulse la causa de la reforma de la ley sobre aborto como un modo de diferenciarse de sus oponentes políticos, y en particular de una presidencia cuya legitimidad se cuestionaba.

La nueva legislación de la Ciudad en materia de aborto fue notable no sólo por su contenido, sino también por su implementación, que se ha evaluado como exitosa en al menos tres aspectos principales: a) por su aplicación rápida y efectiva, así como por la calidad de los servicios prestados (Informante 13, entrevista de la autora 2011; Van Dijk et al., 2011); b) por sus consecuencias para la agencia y el empoderamiento de las mujeres, ya que ha facilitado la apropiación de los derechos reproductivos por muchas mujeres que no los conocían anteriormente (Ortiz-Millán, 2012), y ha inculcado en las mujeres la idea de que su decisión de abortar es legítima (Informante 1, entrevista de la autora 2011); c) por sus efectos en la opinión pública sobre el derecho al aborto (Wilson et al., 2011). De hecho, se ha considerado que la reforma ha provocado un "cambio de paradigma" en la provisión de servicios, solo comparable, dentro del contexto americano, al tipo de servicio ofrecido en Canadá (Informante 13, entrevista de la autora 2011).

En cuanto a las razones relacionadas con el éxito de la implementación, se han destacado varios factores. En primer lugar, el compromiso del Ejecutivo de la Ciudad de México, especialmente Ebrad y el Secretario de Salud, y la colaboración entre el secretario de salud y las ONG han sido fundamentales para la implementación (Informante 13, entrevista de la autora 2011). 
En particular, IPAS-México, una de las cinco organizaciones que forman la Alianza Nacional por el Derecho a Decidir, ha trabajado durante varios años para ayudar a las instituciones de salud pública a brindar servicios de aborto, en coordinación con el secretario de salud (Informante 13, entrevista de la autora 2011). Un factor importante para el éxito en la implementación de esta reforma fue el trabajo previo realizado por el gobierno de la Ciudad de México en la implementación de los servicios de aborto legal desde la reforma de 2000, y su estrecha colaboración desde entonces con las ONG que trabajan en salud reproductiva. Finalmente, la amplitud de la discusión pública sobre el aborto durante el proceso de reforma y las acciones de sensibilización de las instituciones de salud contribuyeron significativamente a una implementación exitosa (Informante 13, entrevista de la autora 2011).

Esta reforma y su implementación en la Ciudad de México pueden entenderse en el marco de la profunda tradición laicista del Estado mexicano. A diferencia de otros países de la región, uno de los aspectos distintivos en el desarrollo del Estado mexicano fue su profundo proceso de secularización, sólo similar al caso uruguayo (Blancarte, 2008b). También fue en la Ciudad de México y en Uruguay donde más tempranamente en la región las legislaturas modificaron las leyes de aborto y legalizaron la práctica. En México, la separación entre el Estado y la Iglesia fue parte del desarrollo político y cultural del país desde mediados del siglo XIX, y se manifestó en disposiciones constitucionales y acuerdos culturales compartidos por amplios sectores sociales, más allá de la religiosidad de la población (Blancarte, 2008a). ${ }^{4}$ La Revolución Mexicana que comenzó en 1910 y la Constitución de 1917 que resultó de ese proceso fueron hitos clave en ese desarrollo. Tuvieron como uno de sus objetivos despojar a la esfera sociopolítica del poder religioso y, de hecho, hasta avanzada la transición democrática en el país, proscribieron a la Iglesia del espacio público (Lamas, 1997: 61; Blancarte, 2008a: 35-37).

$\mathrm{Al}$ considerar un proceso de reforma a nivel subnacional, en la Ciudad de México, es necesario aclarar, también que, si bien las relaciones formales entre la iglesia y el estado son una variable del nivel nacional, regulada por disposiciones constitucionales y leyes federales, existen diferencias subnacionales en términos de reglas informales y de la influencia de facto de la Iglesia Católica y de otros grupos religiosos sobre la política local.

Según el censo nacional de 2010, el 83,9\% de la población se identificó como católica. Sin embargo, la religiosidad de la población se combina con una postura anticlerical, que se ha afirmado que es parte de la identidad nacional de México, y no sólo una cuestión de elites liberales (Blancarte, 2001: 844-845). 
Analistas y activistas coinciden en señalar que en algunos estados del país las fuerzas conservadoras tienen más influencia sobre las élites locales y la política, en comparación, por ejemplo, con la fuerte presencia de actores e instituciones liberales en la Ciudad de México y en la esfera política nacional (Informante 4 y 7, entrevistas de la autora 2011; Informante 12, entrevista de la autora 2019). En este sentido, se observa que existen muchas más violaciones al estado laico a nivel estatal que nacional, y que en el nivel subnacional, fuera de la Ciudad de México, hay más legitimidad de los actores religiosos y una mayor interacción y cercanía con el poder político (Informante 9, entrevista de la autora 2019). Estas diferencias son significativas para analizar las diferencias en la regulación del aborto a lo largo del país, y para explicar también el proceso de contra-reformas que ocurrió a partir de 2008 en diecisiete estados mexicanos que incluyeron en sus constituciones una cláusula de protección a la vida desde la concepción (sobre estas reformas, ver Lopreite, 2014 y Beer, 2017).

\section{Brasil: Política electoral y bloqueo de la posibilidad de reformas a las leyes de aborto}

En Brasil, durante el gobierno del Partido de los Trabajadores (PT), no hubo una oposición ideológica al tema de la legalización del aborto, ni a la implementación del aborto legal, por parte de los Presidentes Luis Ignácio Lula da Silva (2003-2011) y Dilma Rousseff (2011-2016). En realidad, si bien desde su creación, en 1980, el PT tuvo claros vínculos con sectores de la Iglesia Católica (Cleary, 1997: 269), en sus manifiestos se puede encontrar la legalización del aborto como una prioridad programática, ${ }^{5}$ y en términos numéricos, este partido ha sido el impulsor más importante de la liberalización de las leyes de aborto en el Congreso. ${ }^{6}$ Sin embargo, bajo los gobiernos de Lula da Silva y Roussseff no se modificó la ley de aborto, que es una de las más restrictivas del mundo, ya que desde 1940 el aborto es legal sólo en casos de riesgo de vida, violación y malformación fetal, y no se incluye una excepción por riesgo para la salud de la mujer. La inacción del Poder Ejecutivo para impulsar un cambio legal puede explicarse debido un

Por ejemplo, entre las principales resoluciones del tercer Congreso Nacional del PT en 2007, se encontraba "la defensa de la auto-determinación de las mujeres y la descriminalización del aborto y la provisión de su acceso en los servicios públicos de salud".

$6 \quad$ En la década de 1990, el PT fue el partido que presentó más proyectos de ley favorables a la reforma de la ley del aborto (9 de un total de 21) (Casanova, 2000: 72). 
fuerte bloqueo por parte de fuerzas conservadoras, especialmente durante periodos electorales, y dirigida con mayor fuerza hacia la candidata y luego Presidenta Rousseff.

El ascenso del PT al poder nacional en 2003 creó una nueva oportunidad política para promover las demandas del movimiento feminista y de los movimientos sociales en general. Al inicio del primer mandato del presidente Lula da Silva, hubo una incorporación masiva de miembros de los movimientos sociales, incluyendo de manera prominente al feminismo, a la estructura gubernamental, y una integrante de este movimiento fue designada para dirigir el Área de Salud de la Mujer del Ministerio de Salud. Asimismo, durante su gobierno se implementó una amplia estrategia nacional de combate a la mortalidad materna, con la participación de la Red Nacional Feminista por los Derechos y la Salud Sexual y Reproductiva (Redesaúde) (Informante 11, entrevista de la autora 2012). Además, por primera vez un Ministro de Salud brasileño se pronunció a favor de cambiar la ley del aborto (Galli y Sydow, 2009).

Bajo el gobierno de Lula da Silva, en la I Conferencia Nacional de Políticas para la Mujer de 2004, organizaciones sociales recomendaron al gobierno modificar la ley de aborto. El gobierno incorporó este reclamo como una de las seis prioridades en el área de la salud de la mujer, y creó la denominada Comisión Tripartita para la revisión de la legislación sobre interrupción voluntaria del embarazo (Informante 6, entrevista de la autora 2012). Sin embargo, durante sus dos mandatos no se cambió la ley del aborto, y en dos instancias cruciales el gobierno echó atrás sus iniciativas de reforma, debido a la presión de grupos religiosos. Una de estas ocasiones clave involucró el proyecto de la Comisión Tripartita, que finalmente perdió el apoyo del gobierno debido a la presión conservadora y fue derrotado en el Congreso (Informante 3, entrevista de la autora 2012). El otro momento clave fue la exclusión de la declaración del aborto como derecho humano en el III Programa Nacional de Derechos Humanos, en 2010. Estudiosas del tema aseguran que lo que estaba en juego para el gobierno en esa ocasión era la creación de una comisión de la verdad y de reparaciones por los crímenes de la dictadura militar, y la cuestión del aborto se convirtió en moneda de cambio en esa negociación, frente a la oposición religiosa conservadora (Corrêa, 2010: 91; Pitanguy, 2011: 42).

Pero la ofensiva más importante contra cualquier intento de cambio en este campo, incluida la implementación del aborto legal, se produjo durante la campaña presidencial y el gobierno de Dilma Rousseff, y está vinculada con la política del proceso de destitución en su contra. En enero de 2011, Rousseff asumió el cargo como la primera mujer Presidenta de Brasil. Al 
comenzar su mandato, nombró más ministras en su gabinete que nunca antes en este país, y en 2012 designó a Eleonora Menicucci, reconocida académica feminista en el campo de la salud pública y activista por el derecho al aborto, como Ministra de la Secretaría de Políticas para la Mujer. Asimismo, antes de la campaña presidencial, Rousseff había expresado públicamente su apoyo a la legalización del aborto por motivos de salud pública. $^{7}$ Sin embargo, durante la campaña, y en particular durante la segunda vuelta de las elecciones presidenciales, el tema del aborto, que ya era un elemento importante en la política electoral brasileña reciente, se radicalizó debido a las presiones de las iglesias católica y evangélica, y se convirtió en el tema más crucial de la contienda electoral (Corrêa, 2010: 93; Pitanguy, 2011: 42). El principal candidato opositor, el ex Ministro de Salud José Serra, del Partido de la Social Democracia Brasileña (PSDB), cambió su anterior posición progresista y adoptó una posición abierta contra el aborto. En ese contexto, durante la segunda vuelta, y ante la presión de los líderes religiosos, Rousseff firmó un comunicado público comprometiéndose a no tomar la iniciativa para promover cambios en la ley del aborto o en cualquier otro tema relacionado con la familia. ${ }^{8}$

La posición de la Presidenta Rousseff debe entenderse en el contexto de la ascensión y alianza de fuerzas conservadoras en el campo político, particularmente en el Congreso Nacional. Este proceso aún no se había materializado plenamente durante el primer mandato de Lula da Silva, e influyó negativamente en las oportunidades políticas para cualquier cambio por parte del Congreso o el Ejecutivo en materia de derechos sexuales y reproductivos (Informante 5, entrevista de la autora 2012). El crecimiento del peso relativo de actores religiosos en el proceso político, y su influencia especialmente en la política electoral, ha sido crucial para el bloqueo de iniciativas presidenciales progresistas en este campo. Esto se sumó a los obstáculos que enfrentó la Presidencia para la toma de decisiones durante los gobiernos del PT, generados por el hecho de carecer de una mayoría legislativa propia -como sí tuvo el Jefe de Gobierno en la Ciudad de México-,${ }^{9}$ y a las dificultades que presentó en este sentido el presidencialismo de coalición brasileño en el marco de un sistema de partidos híper fragmentado (ver Rezende y Goldestein, 2016).

El marco constitucional brasileño tiene disposiciones importantes para garantizar el laicismo (art. 5, inc. VI, que garantiza la libertad religiosa, y el

Entrevista a Dilma Rousseff, Marie Claire, abril 2009.

El texto de la carta puede encontrarse en: http://media.folha.uol.com.br/poder/2010/ 10/15/carta_mensagem_dilma.pdf.

$9 \quad$ Agradezco esta observación a una evaluación anónima de este trabajo. 
art. 19, inc. I, que prohíbe el financiamiento público de los cultos religiosos). Sin embargo, la educación religiosa está permitida en las escuelas públicas, los símbolos religiosos están presentes en la sede de los poderes del Estado, incluido el Congreso y el Supremo Tribunal Federal), la Iglesia Católica ejecuta proyectos sociales, especialmente para niños, con apoyo de fondos públicos; y los obispos y pastores pueden ocupar cargos oficiales, por ejemplo, como jefes de un ministerio federal o como legisladores. De hecho, la presencia de congresistas religiosos que dedican sus mandatos a promover los intereses y agendas particulares de sus comunidades morales y religiosas es aceptada sin mucha controversia (Diniz y Vélez, 2008: 2-3). Asimismo, a diferencia de lo que ocurre en México, la separación entre las esferas del Estado y las iglesias no ha representado un problema para sectores significativos de la población (Informante 14, entrevista de la autora 2012).

El epicentro de la movilización conservadora y el baluarte de los opositores al aborto en el país es la Cámara Federal de Diputados. La Iglesia Católica ha sido un opositor tradicional del derecho al aborto en Brasil, y ya en 1949, promovió un proyecto legislativo destinado a eliminar las dos excepciones a la criminalización del aborto en el código penal (Casanova, 2000: 71). Además de esta relevancia temprana del catolicismo en este campo, Brasil se ha convertido en el ejemplo más notable de la creciente influencia de las iglesias evangélicas, en su mayoría neopentecostales, en la política de la región. La Iglesia Universal brasileña (Igreja Universal do Reino de Deus), la principal iglesia neopentecostal del país, eligió su primer diputado federal tan pronto como en 1986. Desde entonces, su influencia en el Congreso Nacional, junto a Assambleia de Deus y otros grupos evangélicos, no ha dejado de crecer (Almeida, 2017; Informante 2, entrevista de la autora 2019). Los dos aspectos distintivos de la movilización conservadora en Brasil son su participación directa en el proceso legislativo y la coalición entre actores católicos y evangélicos en el Congreso, formando una bancada religiosa. Esto ha creado una oportunidad política cada vez más favorable para la oposición de los conservadores al derecho al aborto y para su intervención en el proceso político del país en general.

Más allá de su incidencia en el campo de los derechos sexuales y reproductivos como una bancada religiosa prácticamente de tema único, en su oposición a los derechos sexuales y reproductivos, este bloque religioso conservador en el Congreso ha sido un actor crucial en el proceso que condujo a la destitución de la presidenta Rousseff y al ascenso de la extrema derecha al gobierno nacional. El tema del aborto fue un elemento central en la ofensiva contra el Partido de los Trabajadores (PT) y en el giro conservador de la política brasileña. 
El gobierno de Rousseff fue leal a su compromiso de campaña de no modificar las leyes de aborto, al tiempo que afirmó su voluntad de garantizar el acceso al aborto legal y la reducción de riesgos en casos de abortos inseguros (Informante 11, entrevista de la autora 2012). Fue precisamente en torno a la regulación sobre implementación de los abortos legales que, en 2014, se intensificó el conflicto entre el gobierno y los diputados religiosos, por la promulgación de una orden ejecutiva (Portaria 415) destinada a regular el acceso a los abortos legales en el sistema público de salud (Coutinho, 2019). La exitosa oposición a esta medida, que hizo que el gobierno derogara esa medida siete días después de su promulgación, fue liderada por Eduardo Cunha, entonces líder del PMDB (Partido del Movimiento Democrático Brasileño) en la Cámara de Diputados. Esta victoria otorgó a Cunha un papel destacado en la conducción de la bancada evangélica y le permitió convertirse en presidente de la Cámara en 2015, derrotando al candidato del PT en la primera vuelta (Coutinho, 2019). Desde ese cargo, Cunha encabezó el golpe parlamentario contra la presidenta Rousseff en 2015-2016, que condujo a la presidencia provisional de Michel Temer (ver Limongi, 2015) y finalmente a la elección de Jair Bolsonaro en 2018.

\section{Conclusiones}

A través del estudio de los casos de Ciudad de México y Brasil, este artículo analizó cómo razones estratégicas vinculadas al ciclo electoral pueden incidir en la decisión de Ejecutivos de izquierda o centro-izquierda de promover o no avances en materia de legislación e implementación del derecho al aborto. El análisis de los cálculos electorales contribuye a explicar por qué gobiernos de signo progresista difieren en la regulación del aborto, e incluso candidatos de izquierda podrían apoyar mayores restricciones, como ocurrió en los casos de Nicaragua o El Salvador, dada la presión de poderes de facto como la Iglesia.

En los dos casos estudiados, los partidos en el gobierno eran programáticamente afines al avance de los derechos reproductivos, y los líderes que encabezaban el Ejecutivo no manifestaban convicciones personales contrarias a la liberalización del aborto. Sin embargo, mientras que en la Ciudad de México la polarización política en el contexto post-electoral de 2006 contribuyó a generar incentivos para que el gobierno de la Ciudad encarase una reforma de la ley del aborto como forma de diferenciarse de sus contrincantes y fortalecer su base política, en el caso de Brasil las elecciones de 2010 fueron una coyuntura crítica en la que sectores religiosos 
conservadores lograron influir para bloquear cualquier avance en este campo. Posteriormente, la cuestión del aborto fue un asunto importante también en la configuración de fuerzas que condujo a la destitución de la Presidenta Rousseff.

Asimismo, en este artículo se argumentó que la tradición de laicidad en el caso mexicano, y en particular en la Ciudad de México, y la falta de una tradición de separación profunda de las esferas del Estado y la Iglesia en Brasil, sumada al creciente poder de coaliciones religiosas en el Congreso nacional en este país, contribuyen a explicar los resultados disímiles entre ambos casos. El caso de Brasil muestra cómo la presión de la esfera religiosa sobre el campo político puede influir sobre los cálculos presidenciales y condicionar el margen de acción de líderes progresistas y con convicciones personales afines a las demandas feministas en materia de aborto.

Finalmente, si bien este trabajo se centró en el rol de los poderes ejecutivos y sus incentivos electorales, se asume que, para explicar cambios legislativos en esta área de derechos, se debe evaluar también el rol de otras condiciones político-institucionales para aprobar estas leyes, tales como la conformación de las bancadas legislativas y la acción del feminismo tanto en la sociedad civil como al interior de los Estados. Especialmente, en futuros estudios sobre el rol de los poderes ejecutivos en la política del aborto, se debería analizar el peso de los partidos de izquierda en el legislativo, y el tipo de bancada con que cuenta el Presidente, que puede amplificar la influencia de los factores religiosos, o bien disminuirla. ${ }^{10}$ En este sentido, sería importante estudiar el caso brasileño en cada coyuntura crítica bajo esta perspectiva, así como analizar cómo influye esta dinámica legislativa actualmente en el caso mexicano, bajo un gobierno nacional de signo progresista. Asimismo, este análisis contribuiría a entender cómo fue posible la legalización del aborto en la Argentina, donde no ha habido una profunda separación histórica entre Estado e Iglesia, y frente a la presencia de un Papa de esta nacionalidad.

\section{Referencias bibliográficas}

Almeida, R. (2017). A onda-quebrada - evangélicos e conservadorismo. Cadernos Pagu, 50, 1-27.

Beer, C. (2017). Making Abortion Laws in Mexico: Salience and Autonomy in the Policymaking Process". Comparative Politics, 50, 41-59.

10 Agradezco esta observación a una evaluación anónima de este trabajo. 
Blancarte, R. (2008a). "El porqué de un estado laico". En Blancarte, R. (Ed.), Los retos de la laicidad y la secularización en el mundo contemporáneo. Ciudad de México: El Colegio de México.

Blancarte, R. (2008b). Laicidad y laicismo en América Latina. Estudios Sociológicos, (XXVI) 76, 139-164.

Blancarte, R. (2001). Laicidad y Secularización en México. Estudios Sociológicos, XIX, $57,843-855$.

Blofield, M., C. Ewig y J. Piscopo (2017). The reactive left: gender equality and the Latin American pink tide. Social Politics: International Studies in Gender, State E Society, 24(4), 345-369.

Blofield, M. y C. Ewig (2017). The Left Turn and Abortion Politics in Latin America. Social Politics: International Studies in Gender, State E Society 24(4), 481-510.

Casanova, A. (2000). Abortion in Brazil: Legislation, Reality and Options. Reproductive Health Matters, 8(16), 66-76.

Center for Reproductive Rigths (2014). "Abortion Worldwide: 20 Years of Reform". Briefing Paper. Nueva York.

Cleary, E. (1997). The Brazilian Catholic Church and Church-State Relations: Nation Building, Journal of Church and State, 39(2), 253-272.

Corrêa, S. (2010). Brasil: aborto na linha de frente. Reproductive Health Matters, 18(36), $88-95$.

Cortés, A. (2006). Persecución legal del aborto en la Ciudad de México. Un atentado al derecho a decidir. México DF: GIRE.

Coutinho, A. (2019). "A Câmara dos Deputados e a questão do aborto: tensionamento no século XXI." Faces de Clio, 5(9), 123-144.

Friedman, E. J. y C. Tabbush (2020). "Disputas en la marea rosa: una mirada desde el género y la sexualidad”. En Friedman, E. J., (Ed.), Género, sexualidad e izquierdas latinoamericanas. El reclamo de derechos durante la marea rosa. Buenos Aires: CLACSO, pp. 19-70.

Galli, B. y E. Sydow (2009). "O debate sobre o aborto no Brasil: avanços, retrocessos e perspectivas". Río de Janeiro: Ed. Ações Afirmativas em Direitos e Saúde.

GIRE (2008). "El proceso de despenalización del aborto en la Ciudad de México", Colección "Temas para el debate", No. 7. Ciudad de México: Grupo de Información en Reproducción Elegida (GIRE). Disponible en https://gire.org.mx/wpcontent/uploads/2016/08/ProcesoDespena_TD7.pdf

Grzymała-Busse, A. (2015). Nations under God: How Churches Use Moral Authority to Influence Policy. Princeton: Princeton University Press.

Htun, M. (2003). Sex and the State: Abortion, Divorce, and the Family under Latin American Dictatorships and Democracies. Cambridge: Cambridge University Press.

Htun, M. y L. Weldon (2010). When do governments promote women's rights? A framework for the comparative analysis of sex equality policy. Perspectives on Politics, 8(1), 207-216. 
Johnson, N., A. L. Rodríguez Gustá, y D. Sempol (2020). "Claves para explicar avances y retrocesos en los derechos de las mujeres y las personas LGBT en Uruguay." En Friedman, E. J. (Ed.), Género, sexualidad e izquierdas latinoamericanas. El reclamo de derechos durante la marea rosa. Buenos Aires: CLACSO, pp. 71-108.

Kampwirth, K. (2008). Abortion, antifeminism, and the return of Daniel Ortega:InNicaragua, leftist politics? Latin American Perspectives, 35, 122-36.

Lamas, M. (2017). La interrupción legal del embarazo: El caso de la Ciudad de México. Ciudad de México: Fondo de Cultura Económica.

Lamas, Marta (2009). "La despenalización del aborto en México". Nueva Sociedad, 220. Disponible en https://nuso.org/articulo/la-despenalizacion-del-aborto-enmexico/

Lamas, M. (1997). The Feminist Movement and the Development of Political Discourse on Voluntary Motherhood in Mexico. Reproductive Health Matters, 10, November, 59-67.

Lamas, M. y S. Bissell (2000). Abortion and Politics in Mexico: Context is all, Reproductive Health Matters 8(16), 10-23.

Limongi, F. (2015). O passaporte de cunha e o impeachment a crônica de uma tragédia anunciada. Novos estudos CEBRAP, 103, 99-112.

Lopreite, D. (2020). "The federal restriction of womens rights: Argentina's politics on abortion and contraception.” En Vickers, J., J. Grace y C. Collier (Eds.), Handbook on Gender, Diversity and Federalism. Edward Elgar Publishing, pp. 212-226.

Lopreite, D. (2014). Explaining policy outcomes in federal contexts: the politics of reproductive rights in Argentina and Mexico. Bulletin of Latin American Research 33(4), 389-404.

Mainwaring, S. (1997). "Presidentialism in Brazil: The Impact of Strong Constitutional Powers, and Robust Federalism”, Working Paper No. 225, Woodrow Wilson Center.

Minkenberg, M. (2002). Religion and Public Policy: Institutional, Cultural, and Political Impact on the Shaping of Abortion Policies in Western Democracies. Comparative Political Studies,35(2), 221-47.

Narváez, María Fernanda (2017). "El discurso del Presidente de Ecuador frente al proceso legislativo sobre penalización del aborto en casos de violación”, Tesis de Maestría en Políticas Públicas y Género, FLACSO México.

O'Donnell, G. (2011). "Nuevas reflexiones acerca de la democracia delegativa". En O’Donnell, G.; Iazzeta, O.y Quiroga, H., Democracia Delegativa. Buenos Aires: Prometeo.

Ortiz-Millán, G. (2012). La Moralidad del aborto, México DF: Siglo XXI.

Outshoorn, J. (1996). "The stability of compromise: abortion politics in Western Europe”. En Githens, M. y McBride Stetson, D. (Eds), Abortion politics: Public policy in cross-cultural perspective. Nueva York: Routledge, pp. 145-165. 
Pitanguy, J. (2011). "Advocacy: um processo histórico". En Linhares Barsted , L.y J. Pitanguy, O Progresso das Mulheres no Brasil 2003-2010. Río de Janeiro: CEPIA, ONU Mujeres, pp. 22-56.

Reuterswärd, C., Zetterberg, P., Thapar, S., y M. Molyneux (2011). Abortion Law Reforms in Colombia and Nicaragua: Issue Networks and Opportunity Contexts. Development and Change, 42(3), 805-831.

Rezende, R. y A. Goldstein (2016). Brasil: fin de los gobiernos del PT, presente de crisis y resistencias. Revista Política Latinoamericana, 1-16.

Rubio Marin, R. (2014). "Abortion in Portugal: New Trends in European Abortion Constitutionalism”. En Cook, R. y J. Erdmann (Eds.), Abortion Law in Transnational Perspective: Cases and Controversies. Philadelphia: University of Pennsylvania Press.

Ruibal, A. (2012). Feminismo, religión y democracia en el proceso de legalización del aborto en la ciudad de México. Estudios, 27, 13-26.

Sánchez Fuentes, M. L., Paine, J.y Elliott-Buettner, B. (2008). The decriminalisation of abortion in Mexico City: how did abortion rights become a political priority? Gender and Development, 16(2), 345-360.

Tabbush, C., Díaz,m. C., Trebisacce, C. y V. Keller (2020). "Matrimonio igualitario, identidad de género y disputas por el derecho al aborto en Argentina. La política sexual durante el kirchnerismo (2003-2015)". En Friedman, E. J. (Comp.), Género, sexualidad e izquierdas latinoamericanas. El reclamo de derechos durante la marea rosa. Buenos Aires: CLACSO, pp. 109-142.

Tarrow, S. (1996). "States and opportunities: The political structuring of social movements". En McAdam, D., McCarthy, J. y Zald, M. (Eds.) Comparative Perspectives on Social Movements, Political Opportunities, Mobilizing Structures, and Cultural Framings. Nueva York: Cambridge University Press, pp. 41-61.

Trejo, G. y S. Ley (2016). Federalismo, drogas y violencia. Por qué el conflicto partidista intergubernamental estimuló la violencia del narcotráfico en México. Política y gobierno, 23(1), 11-56.

Van Dijk, M., L. Arellano, A. G. Arangure, A. Alberto Toriz, A. Krumholz y E. Yam (2011). Women's Experiences with Legal Abortion in Mexico City: A Qualitative Study, Studies in Family Planning, 42(3), 167-174.

Viterna, J. (2012). The left and "life" in El Salvador. Politics E Gender 8(2), 248-254. Wilson, K., S. García, C. Díaz, A. Villalobos, J. Valencia, P. Sanhueza y C. Burks (2011). Public Opinion on Abortion in Mexico City after the Landmark Reform. Studies in Family Planning, 42(3), 175-182.

Zaremberg, G. (2016). ¿Género versus pueblo?: Movilización, cooptación y participación en Venezuela, Brasil y Nicaragua. Latin American Research Review, 51 (1), 84108. 


\section{Entrevistas}

Informante 1. Entrevista de la autora. Ciudad de México, 23 de setiembre de 2011. Informante 2. Entrevista de la autora. Brasilia, 23 de agosto de 2019.

Informante 3. Entrevista de la autora. São Paulo, 28 de agosto de 2012.

Informante 4. Entrevista de la autora, Ciudad de México, 13 de setiembre de 2011.

Informante 5. Entrevista de la autora. Brasilia, 15 de agosto de 2012.

Informante 6. Entrevista de la autora. Brasilia, 16 de agosto de 2012.

Informante 7. Entrevista de la autora. Ciudad de México, 20 de setiembre de 2011. Informante 8. Entrevista de la autora. Ciudad de México, 30 de setiembre de 2011. Informante 9. Entrevista de la autora. Ciudad de México, 26 de setiembre de 2019. Informante 10. Entrevista de la autora. Ciudad de México, 7 de setiembre de 2011. Informante 11. Entrevista de la autora. Porto Alegre, 8 de agosto de 2012.

Informante 12. Entrevista de la autora. Ciudad de México, 26 de setiembre de 2019. Informante 13. Entrevista de la autora. Ciudad de México, 29 de setiembre de 2011. Informante 14. Entrevista de la autora. São Paulo, 27 de Agosto 2012. 